\title{
Zero-Curvature Representation of Non-Abelian Quantum Painleve II Equation with Its Darboux Solution
}

\section{Irfan Mahmood*}

CHEP, University of the Punjab, Lahore 54590, Pakistan

\begin{abstract}
In this article a non-abelian quantum analogue of classical Painleve II equation is presented with its zerocurvature condition that involves the quantum Painleve II symmetric form with the quantum commutation relations introduced by Nagoya and a new commutation relation between variable $\mathrm{z}$ and the $\mathrm{f} f(\mathrm{z})$ such as $z f-f z=\frac{1}{i \hbar f}$ is established. Further, the Lax representations to higher order symmetric forms of Painleve IV equation and Painleve $V$ equation are introduced. Finally, non-trivial Darboux solutions of non-abelian quantum Painleve II equation iare derived through its linear representation.
\end{abstract}

Keywords: Zero-curvature condition; Painleve II equation; Darboux transformations

\section{Introduction}

One of the recent development in the theory Painleve equations is their extension quantum and non cummutaive (NC). In that directions it was attempted successfully $[1,2]$ where the quantum analogues of these equations by using their symmetric form these equation presented in a study [3], for example the quantum version of classical Painleve II equation can be obtained on solving the following system for field $\mathrm{f}_{2}$ :

$$
\left\{\begin{array}{c}
f_{0}^{\prime}=f_{o} f_{2}+f_{2} f_{o}+\alpha_{0} \\
f_{1}^{\prime}=-f_{1} f_{2}-f_{2} f_{1}+\alpha_{1} \\
f 2=f 1-f_{0}
\end{array}\right.
$$

Where the fields $f_{0}, f_{1}, f_{2}$ are subjected to obey some quantum commutation relations and $\alpha_{0}, \alpha_{1}$ are constant parameters. The quantum Painleve II equation,

$$
f^{\prime \prime}=2 f_{2}^{3}-2 z f_{2}+c
$$

derived [1] may be considered as matrix version of classical Painleve II equation because the quantum commutation relations are defined for the fields where as the fields $\mathrm{f}_{\mathrm{i}}$ and variable $\mathrm{z}$ are commuting and $c=\alpha_{1} \alpha_{0}$. In classical framework various integrable aspects of Painleve II equation have been studied such as the associated RiemannHilbert problem, connection to well known integrable systems and its Hamiltonian hierarchies detail can be found in early studies [4-6]. The study of its quantum and NC analogues therefore is important because that equation has been taken as s model in many physicals problems, few are mentioned in [7-11]. In order to understand the compatibility of Painleve equation from mathematical and physical point of views it will interesting to study their different properties on noncommutative spaces as other well known integrable systems [12-24] possess on deformed spaces.Keeping that motivation an initial achievement in NC direction was obtained by Retakh and Roubtsov [25], where they have introduced purely NC version of PII equation,

$$
f_{2}^{\prime \prime}=2 f_{2}^{3}-2\left[z, f_{2}\right]_{+}+4\left(\beta+\frac{1}{2}\right)
$$

by using its symmetric form presented in previous study [1] and in their computations the fields $\mathrm{f}_{0}, \mathrm{f}_{1}, \mathrm{f}_{2}$ and variable $\mathrm{z}$ obey a kind of star product, purely non-commuting elements and more over its solutions were expressed in terms of to NC Toda solutions. One the contribution in that direction can be found in a study [26] where quasideterminant solutions of that NC PII equation presented through the Darboux transformations. Later on the zero-curvature representation of associated nonlinear equations to NC Toda systems of a study [25] obtained previously [27] and further these results were extended to calculate NC PII solutions taking NC Toda solutions as seed at $n=0$ in its Darboux solutions.

In this article we have extended the Nagoya [1] work on quantum PII equation to derive it non-abelian analogue. Here I have introduced a zero-curvature condition that is equivalent to the non-abelian quantum PII equation,

$$
\left\{\begin{array}{c}
f^{\prime \prime}=2 f_{2}^{3}-2\left[z, f_{2}\right]_{+}+c \\
z f_{2}-f_{2} z=\frac{1}{2} i \hbar f_{2}
\end{array}\right.
$$

these derivations are also involved the symmetric form (1) of PII equation and fields $f_{0}, f_{1}, f_{2}$ obey the quantum commutation relation given in a study [1].The basic difference between the quantum PiI equation and the non-abelian quantum PII eqn. (4) is that here the variable $\mathrm{z}$ and field $\mathrm{f}_{2}$ appear as non commuting elements but in cases of Nagoya [1] these elements treated classically, as commuting variables. Further this can be shown that under the classical limit $\hbar \rightarrow 0$ the system (4) reduces to its classical analogue. More over the non-trivial Darboux solutions of Non-abelian quantum PII with its riccati form are presented.

\section{Lax Formalism and Zero-Curvature Condition}

The Lax formalism first was introduced by Lax [28] that plays very important role in theory of integrable whose compatibility condition yields Lax equation. For given two operators, say Land $\mathrm{P}$, are subjected to a linear system $\mathrm{L}(\mathrm{x}, \mathrm{t}) \Psi=\lambda \Psi, \Psi \mathrm{t}=\mathrm{P}(\mathrm{x}, \mathrm{t}) \Psi$ and the compatibility condition yields Lax equation,

$$
\mathrm{L}_{\mathrm{t}}=[\mathrm{P}, \mathrm{L}]
$$

Where $\Psi$ is a column vector, $\lambda$ is spectral parameter and $[\mathrm{P}, \mathrm{L}]$

*Corresponding author: Irfan Mahmood, CHEP, University of the Punjab Lahore 54590, Pakistan, Tel: 0092-55-9201222; E-mail: mahirfan@yahoo.com

Received March 29, 2018; Accepted June 27, 2018; Published July 08, 2018

Citation: Mahmood I (2018) Zero-Curvature Representation of Non-Abelian Quantum Painleve II Equation with Its Darboux Solution. J Phys Math 9: 276. doi: 10.4172/2090-0902.1000276

Copyright: (c) 2018 Mahmood I. This is an open-access article distributed unde the terms of the Creative Commons Attribution License, which permits unrestricted use, distribution, and reproduction in any medium, provided the original author and source are credited. 
is commutator. The Lax formalism extensively has been applied to study various integrable aspects of classical as well as NC integrable systems such as to construct their solitonic solution through the inverse scattering data and one of interesting use of Lax systems is to derive Darboux-Backlund transformations of integrable systems. More over many other properties of integrable systems has been studied in wide spectrum in the framework Lax formalism, see for example [1219]. The Lax representations to the symmetric forms of higher order Painleve equations mentioned in propositions 1.1 and 1.2. Further this can be calculated that linear system $\Psi_{\mathrm{x}}=\mathrm{A}(\mathrm{x}, \mathrm{t}) \Psi$ and $\Psi_{\mathrm{t}}=\mathrm{B}(\mathrm{x}, \mathrm{t}) \Psi$ is equivalent to expression,

$$
\mathrm{A}_{\mathrm{t}}-\mathrm{B}_{\mathrm{x}}=[\mathrm{B}, \mathrm{A}]
$$

called zero curvature which has been applied to many classical and NC systems, for a brief description [20-24]

\section{Proposition 1.1}

The 1 symmetric form presented in,

$$
\left\{\begin{array}{l}
f_{0}^{\prime}=f_{0} f_{1}-f_{2} f_{0}+\alpha_{0} \\
f_{1}^{\prime}=-f_{1} f_{2}-f_{0} f_{1}+\alpha_{1} \\
f_{1}^{\prime}=-f_{2} f_{0}-f_{1} f_{2}+\alpha_{2}
\end{array}\right.
$$

to quantum PIV equation can be represented by a Lax equation.

Proof: From above systems quantum PIV equation can be obtained by eliminating $\mathrm{f}_{0}$ and $\mathrm{f}_{2}$ from the system (7) here the dependent variable $\mathrm{f}_{0}, \mathrm{f}_{1}$ and $\mathrm{f}_{2}$ obey the following relation,

$$
\partial \mathrm{t}\left(\mathrm{f}_{0}+\mathrm{f}_{1}+\mathrm{f}_{2}{ }_{2}\right)=\mathrm{k} \text {, }
$$

Where $\mathrm{k}=\mathrm{a}_{0}+\alpha_{1}+\alpha_{2}$ for simplicity $\mathrm{k}$ is normalized to 1 .This system also admit the affine Weyl group actions of type A(1) l, see the detail in section 3:3. For the Lax representation to symmetric form of PIV eqn. (7) let us define the diagonal elements of mtrices L and P in eqn. (5) by,

$$
L_{i}=\left(\begin{array}{ll}
1 & 0 \\
f_{i} & 1
\end{array}\right)
$$

Where $\mathrm{i}=0,1,2$

$$
P_{o}=\left(\begin{array}{cc}
-f_{1} & 0 \\
0 & \Gamma_{0}
\end{array}\right), P_{1}=\left(\begin{array}{cc}
-f_{2} & 0 \\
0 & \Gamma_{1}
\end{array}\right), P_{2}=\left(\begin{array}{cc}
-f_{0} & 0 \\
0 & \Gamma_{2}
\end{array}\right)
$$

Respectively, where,

$$
\Gamma_{0}=-f_{2}+\alpha 0 f_{0}^{-1}, \Gamma_{1}=-f_{0}+\alpha_{1} f_{1}^{-1} \Gamma_{2}=-f_{1}+\alpha_{2} f_{2}^{-1}
$$

We can show that the Lax eqn. (5) for the operators A and B with diagonal elements defined above yields the system system (7).

\section{Proposition 1.2}

The generating system of quantum PV equation,

$$
\left\{\begin{array}{l}
f_{0}^{\prime}=f_{0} f_{1} f_{2}-f_{2} f_{3} f_{0}+\left(\frac{1}{2}-\alpha_{2}\right) f_{0}+\alpha_{0} f_{2} \\
f_{1}^{\prime}=-f_{1} f_{2} f_{3}-f_{3} f_{0} f_{1}+\left(\frac{1}{2}-\alpha_{3}\right) f_{1}+\alpha_{1} f_{3} \\
f_{2}^{\prime}=f_{2} f_{3} f_{0}-f_{0} f_{1} f_{2}+\left(\frac{1}{2}-\alpha_{0}\right) f_{2}+\alpha_{2} f \\
f_{3}^{\prime}=f_{3} f_{0} f_{1}-f_{1} f_{2} f_{3}+\left(\frac{1}{2}-\alpha_{2}\right) f_{3}+\alpha_{3} f
\end{array}\right.
$$

Can be written in term of Lax operator.

Proof: In above systems $(8) \mathrm{f}_{0}, \mathrm{f}_{1}, \mathrm{f}_{2}, \mathrm{f}_{3}$ are NC functions of $\mathrm{z}$ and $\alpha_{0}$, $\alpha_{1}, \alpha_{2}, \alpha_{3}$ are parameters this systems also obey the affine Weyl group actions. The dependent functions $f_{0}, f_{1}, f_{2}, f_{3}$ are further subjected to the remarks $f_{0}^{\prime}+f_{2}^{\prime}=\frac{f_{0}+f_{2}}{2}$ and $f_{1}^{\prime}+f_{3}^{\prime}=\frac{f_{1}+f_{3}}{2}$ then by introducing time variable explicitly through the exponential $e^{\frac{z}{2}}$ two of the variables can be easily eliminated. Lets introduce an auxiliary variable $\omega=1-\frac{e^{\frac{z}{2}}}{f_{0}}$ and solve the system (8) for $\omega$ we obtain quantum PV equation see details in section 3.3 Now let us define Lax operators $L$ and $L$ as block matrices of order 4 , as under,

$$
L=\left(\begin{array}{cccc}
L_{0} & \mathrm{O} & \mathrm{O} & \mathrm{O} \\
\mathrm{O} & L_{1} & \mathrm{O} & \mathrm{O} \\
\mathrm{O} & \mathrm{O} & L_{2} & \mathrm{O} \\
\mathrm{O} & \mathrm{O} & \mathrm{O} & L_{3}
\end{array}\right)
$$

With diagonal element $L_{i}=\left(\begin{array}{cc}1 & 0 \\ -f_{i} & -1\end{array}\right)$

The second Lax operator can be written as,

$$
P=\left(\begin{array}{llll}
P_{0} & \mathrm{O} & \mathrm{O} & \mathrm{O} \\
\mathrm{O} & P_{1} & \mathrm{O} & \mathrm{O} \\
\mathrm{O} & \mathrm{O} & P_{2} & \mathrm{O} \\
\mathrm{O} & \mathrm{O} & \mathrm{O} & P_{3}
\end{array}\right)
$$

And element of this operator is given by:

$$
\begin{aligned}
& P_{0}=\left(\begin{array}{cc}
\Theta_{0} & 0 \\
0 & -f_{2} f_{3}
\end{array}\right), P_{1}=\left(\begin{array}{cc}
\Theta_{3} & 0 \\
0 & -f_{3} f_{0}
\end{array}\right) \\
& P_{2}=\left(\begin{array}{cc}
\Theta_{2} & 0 \\
0 & -f_{0} f_{1}
\end{array}\right), P_{3}=\left(\begin{array}{cc}
\Theta_{3} & 0 \\
0 & -f_{1} f_{2}
\end{array}\right)
\end{aligned}
$$

Where,

$$
\begin{aligned}
& \Theta_{0}=-f_{1} f_{2}-\left(\frac{1}{2}-\alpha_{2}\right)-f_{0}^{-1} \alpha_{0} f_{2} \\
& \Theta_{1}=-f 2 f 3-\left(\frac{1}{2}-\alpha_{3}\right)-f_{1}^{-1} \alpha_{1} f_{3} \\
& \Theta_{2}=-f_{3} f_{0}-\left(\frac{1}{2}-\alpha_{0}\right)-f_{2}^{-1} \alpha_{2} f_{0} \\
& \Theta_{3}=-f_{0} f_{1}-\left(\frac{1}{2}-\alpha_{1}\right)-f_{3}^{-1} \alpha_{3} f_{1}
\end{aligned}
$$

If we compute Lax eqn. (5) for the operators (18) and (19) we obtain quantum PV systems. 3. Zero-curvature representation of nonabelian QPII equation.

\section{Zero-Curvature Representation of Nonabelian QPII Equation}

\section{Proposition 1.3}

The following linear system:

$\Psi_{\lambda}=A(z ; \lambda) \Psi, \Psi=B(z ; \lambda) \Psi$

With lax matrics,

$$
\left\{\begin{array}{c}
A=\left(8 i \lambda^{2}+i f_{2}^{2}-2 i z\right) \sigma_{3}+f_{2}^{\prime} \sigma_{2}+\left(\frac{1}{4} c \lambda^{-1}-4 \lambda f_{2}\right) \sigma_{1}+i \hbar \sigma_{2} \\
B=-2 i \lambda \sigma_{3}+f_{2} \sigma_{1}+f_{2} I
\end{array}\right.
$$

yields non-abelian quantum PII equation with quantum commutation 
relations given in a study [1].

Proof: Now starting from following condition,

$$
\mathrm{A}_{\mathrm{z}}-\mathrm{B}_{\lambda}=[\mathrm{B}, \mathrm{A}]
$$

And linear system (12) we can calculate the following values,

$A_{z}=\left(i f_{2}^{\prime} f_{2}+i f_{2} f_{2}^{\prime}-2 i\right) \sigma_{3}+f_{2}^{\prime \prime} \sigma_{2}-4 \lambda f_{2}^{\prime} \sigma_{1}$

$\mathrm{B} \lambda=-2 \mathrm{i} \sigma_{3}$

And,

$$
[B, A]=\left(\begin{array}{cc}
i f_{2}^{\prime} f_{2}+i f_{2} f_{2}^{\prime}+\left[f_{2}, z\right]_{-}-i \hbar & \delta^{+} \\
\delta^{-} & -i f_{2}^{\prime} f_{2}-i f_{2} f_{2}^{\prime}\left[z, f_{2}\right]_{-}+i \hbar
\end{array}\right)
$$

Where,

$$
\delta^{+}=-i f_{2}^{\prime \prime}+2 i f_{2}^{3}-2 i\left[z, f_{2}\right]_{+}+i c+i\left[f_{2}^{\prime}, f_{2}\right]_{-}+4 i \lambda \hbar
$$

and

$$
\delta^{-}=i f_{2}^{\prime \prime}-2 i f_{2}^{3}+2 i\left[z, f_{2}\right]_{+}-i c+i\left[f_{2}, f_{2}^{\prime}\right]_{-}-4 i \lambda \hbar
$$

Above result with eqn. (13) are equivalent to,

$$
\left(\begin{array}{cc}
{\left[f_{2}, z\right]_{-}-\frac{1}{2} i \hbar} & \delta^{+} \\
\delta^{-} & {\left[z, f_{2}\right]_{-}+\frac{1}{2} i \hbar}
\end{array}\right)=0
$$

And we have,

$$
\left[f_{2}, z\right]=\frac{1}{2} i \hbar f_{2}
$$

And

$$
i f_{2}^{\prime \prime}-2 i f_{2}^{3}+2 i\left[z, f_{2}\right]_{+}-i c+i\left[f_{2}, f_{2}^{\prime}\right]_{-}-4
$$

Eqn. (18) is a new result associated to quantum P-II equation. In eqn. (19)the term $i\left[f_{2}^{\prime}, f_{2}^{\prime}\right]_{-}-2 i \lambda \hbar$ can be eliminated by using equation $f_{2}^{\prime}=f_{1}-f_{0}$ from eqn. (1) the quantum commutation relation in eqn. (2) and row replace $\mathrm{f}_{2}$ by $-\frac{1}{2} \lambda^{-1} f_{2}$, then commutation relation become,

$$
\left[f_{0}, f_{2}\right]_{-}=\left[f_{2}, f_{1}\right]_{-}=-2 \lambda \hbar
$$

Now we can write following commutation relation,

$$
\left[f_{2}^{\prime}, f_{2}\right]_{-}=\left[f_{1}, f_{2}\right]_{-}=-\left[f_{0}, f_{2}\right]_{-}
$$

And then applying (20) we get,

$$
\left[f_{2}^{\prime}, f_{2}\right]_{-}=-4 \lambda \hbar
$$

Now after substituting the value of $\left[f_{2}^{\prime}, f_{2}\right]_{-}$from (21) in (19) we get, $f_{2}^{\prime \prime}-2 i f_{2}^{3}+2 i\left[z, f_{2}\right]_{+}-i c=0$

And after using (11) we find the following relation,

$$
\left\{\begin{array}{c}
f_{2}^{\prime \prime}=2 f_{2}^{3}-2\left[z, f_{2}\right]_{+}+c \\
z f_{2}-f_{2} z=\frac{1}{2} i \hbar f_{2}
\end{array}\right.
$$

System (22) can be regarded as nonabelian quantum P II equation and its classical analogue can be obtained as $\hbar \rightarrow 0$

\section{Darboux Transformation for Non-Abelian Quantum PII Equation}

\section{Proposition 2.1}

The Darboux transformation for the solution $\mathrm{u}$ of non-abelian quantum PII eqn. (22) with the help of its associated linear system can be constructed in the following form

$$
u[1]=-4 \lambda \Phi_{1} \chi^{-1}+\Phi_{1} \chi_{1}^{-1} u \Phi_{1} \chi_{1}^{-1}
$$

here $\mathrm{u}[1]$ is a new solution of QP-II equation generated by initial solution $\mathrm{u}$, here $\mathrm{f}_{2}$ has been replaced by $\mathrm{u}$, just for a simple notation.

Proof: For the derivation of non-abelian QP-II Darboux transformation we consider the linear system $(11)$ and $\psi=\left(\begin{array}{l}\chi \\ \Phi\end{array}\right)$, we
have,

$$
\begin{aligned}
& \left(\begin{array}{l}
\chi \\
\Phi
\end{array}\right)_{\lambda}=\left(\begin{array}{cc}
8 i \lambda^{2}+i u^{2}-2 i z & -i u_{z}+\frac{1}{4} C \lambda^{-1}-4 \lambda u+\hbar \\
i u_{z}+\frac{1}{4} c \lambda^{-1}-4 \lambda u-\hbar & -8 i \lambda^{2}-i u^{2}+2 i z
\end{array}\right)\left(\begin{array}{l}
\chi \\
\Phi
\end{array}\right) \\
& \left(\begin{array}{l}
\chi \\
\Phi
\end{array}\right)=\left(\begin{array}{cc}
-2 i \lambda+u & u \\
u & 2 i \lambda+u
\end{array}\right)\left(\begin{array}{l}
\chi \\
\Phi
\end{array}\right)
\end{aligned}
$$

The standard transformation [29] on components of vector $\Psi$ are,

$$
\begin{aligned}
& \chi \rightarrow \chi[1]=\lambda \Phi-\lambda_{1} \Phi_{1}\left(\lambda_{1}\right) \chi_{1}^{-1}\left(\lambda_{1}\right) \chi \\
& \Phi=\Phi[1]=\lambda \chi-\lambda_{1} \chi_{1}\left(\lambda_{1}\right) \Phi_{1}^{-1}\left(\lambda_{1}\right) \Phi
\end{aligned}
$$

Solution at $\lambda$ and $\chi 1(\lambda 1), \Phi 1(\lambda 1)$ with solution at $\lambda=\lambda 1$ of equations and (24) and with eqns. (25) and (26), we have,

$$
\left(\begin{array}{l}
\chi[1] \\
\Phi[1]
\end{array}\right)=\left(\begin{array}{cc}
8 i \lambda^{2}+i u^{2}[1]-2 i z & b_{+} \\
b_{-} & -8 i \lambda^{2}-i u^{2}[1]+2 i z
\end{array}\right)
$$

Where,

$$
\begin{aligned}
& b_{+}=-i u_{z}[1]+\frac{1}{4} C \lambda^{-1}-4 \lambda u[1]+\hbar \\
& b_{-}=i u_{z}[1]+\frac{1}{4} C \lambda^{-1}-4 \lambda u[1]+\hbar \\
& \left(\begin{array}{l}
\chi[1] \\
\Phi[1]
\end{array}\right)=\left(\begin{array}{cc}
-2 i \lambda+u[1] & u[1] \\
u[1] & 2 i \lambda+u[1]
\end{array}\right)\left(\begin{array}{l}
\chi[1] \\
\Phi[1]
\end{array}\right)
\end{aligned}
$$

By using eqns. (24) and (28) we get,

$$
\begin{aligned}
& \chi_{z}=(-2 i \lambda+u) \chi+u \Phi \\
& \Phi_{z}=(i \lambda+u) \Phi+u \chi
\end{aligned}
$$

And

$$
\begin{aligned}
& \chi_{z}[1]=-(2 i \lambda+u[1]) \chi[1]+u[1] \Phi[1] \\
& \Phi_{z}[1]=(2 i \lambda+u[1]) \Phi[1]+u[1] \varphi u[1]
\end{aligned}
$$

And now transformation on $\mathrm{u}$,

$$
u[1]=-4 \lambda \Phi_{1} \chi_{1}^{-1}+\Phi 1 \chi_{1}^{-1} u \Phi_{1} \chi_{1}^{-1}
$$

\section{Conclusion}

In this article a procedure has been detailed to construct the nonabelian analogue of quantum PII equation given in a study [1] and further non-trivial Darboux solutions of that system are also presented. This may be taken as initial step to construct the non-abelian analogues of remaining members of Painleve transcendents involve the quantum commutation relations between variables and fields as new results with their Darboux solutions through linear representations as done in this article for non-abelian quantum PII equation. 
Citation: Mahmood I (2018) Zero-Curvature Representation of Non-Abelian Quantum Painleve II Equation with Its Darboux Solution. J Phys Math 9: 276. doi: 10.4172/2090-0902.1000276

Page 4 of 4

\section{Acknowledgement}

I am thankful to the Punjab University providing me research grant to compete my project.

\section{References}

1. Nagoya H (2004) Quantum Painleve systems of type $A_{t}$. Int J Math 15 : 10071031.

2. Nagoya H, Grammaticos B, Ramani A (2008) Quantum Painleve equations: from Continuous to discrete. SIGMA 4: 051.

3. Noumi M, Yamada $Y$ (1998) Higher order Painleve equations of type $A_{t}$ Funkcial Ekvac 41: 483-503.

4. Fokas AS, Its AR, Kapaev AA, Novokshenov VY (2006) Painleve transcendents: The Riemann-Hilbert approach. Mathematical Surveys and Monographs 128.

5. Joshi $H$ (2004) The second Painleve hierarchy and the stationarty KdV hierarchy. Publ RIMS Kyoto Univ 40: 1039-1061.

6. Joshi H, Mazzocco M (2003) Existence and uniqueness of tritronque solutions of the second Painlev hierarchy. Nonlinearity 16: 427-439.

7. Kudryashov NA (1997) The second Painleve equation as a model for the electric field in a semiconductor. Phys Lett A 233: 397-400.

8. Fokas AS, Tanveer A (1998) A Hele-Shaw problem and second Painleve transcendents. Math Proc Comb Phil Soc 124: 169-191.

9. Jimbo M, MiwaT, Mori X, Sato M (1980) Density matrix of impenetrable bose gas and the fifth Painleve transcendent. Physica D1: 80-158.

10. lyer R, Johnson CV, Pennington JS (2010) String Theory and Water Waves. SLAC-PUB-15670.

11. Barouch E, McCoy BM, Tracy CA, Wu TT (1973) Zero field susceptibility of the two-dimensional using model near Tc. Phys Rev Lett 31: 1409-1411.

12. Dimakis A, Hoissen FM (2000) Noncommutative Kortewegde Vries equation Preprint hep-th/0007074.

13. Kupershmidt BA (1995) Noncommutative integrable systems, in nonlinear evolution equations and dynamical systems, NEEDS 1994. Makhankov V, et al. (eds.) World Scientific 84-101.

14. Okamoto K, Conte R (1999) The Painleve property, One century later. CRM Series in Mathematical Physics. Springer, Berlin 735-787.
15. Balandin SP, Sokolov VV (1998) On the Painleve test for non-abelian equations. Phys Lett A 246: 267-272.

16. Paniak LD (2001) Exact noncommutative KP and KdV multisolutons hep th/0105185

17. Hamanaka M, Toda K (2003) Towards noncommutative integrable systems Phys Lett A 316: 77-83.

18. Hamanaka M, Toda K (2003) Noncommutative Burgers equation. J Phys A 36: 11981.

19. Legare M (2000) Noncommutative generalized NS and super matrix KdV systems from a noncommutative version of (selfdual Yang-Mills equations anti-) Preprint hepth/001207.

20. Dimakis A, Hoissen FM (2007) With a Cole-Hopf transformation to solution of noncommutative KP hierarchy in terms of Wronski matrices. J Phys A 40: F32.

21. Dimakis A, Hoissen FM (2000) The Kortewegde Vries equation on a noncommutative space-time. Phys Lett A 278: 139-145.

22. Camero IC, Moriconi M (2003) Noncommutative integrable field theories in $2 d$ Nucl Phys B 673: 437-454.

23. Gilson CR, Nimmo JJC (2007) On a direct approach to quaside terminant solutions of a noncommutative KP equation. J Phys A 40: 3839-3850.

24. Carillo S, Schieblod C (2009) Noncommutative Kortewegde Vries and modified Kortewegde Vries hierarchies via recursion methods. J Math Phys 50: 073510.

25. Retakh V, Rubtsov V (2010) Noncommutative Toda chain, Hankel quasideterminants and Painleve II equation. J Phys A Math Theor 43: 505204

26. Irfan M (2012) Lax pair representation and Darboux transformation of noncommutative Painleve's second equation. J Geom Phys 62: 1575-1582.

27. Irfan Mahmood (2015) Quasideterminant solutions of NC Painleve II equation with the Toda solution at $n=1$ as a seed solution in its Darboux transformation J Geom Phys 95: 127-136.

28. Lax $P$ (1968) Integrals of nonlinear equations of evolution and solitary waves Commun Pure Appl Math XXI 21: 467-490.

29. Matveev VB, Salle MA (1991) Darboux Transformations and Soliton. Berlin Springer.f 